\title{
A Neuromorphic Design Using Chaotic Mott Memristor with Relaxation Oscillation
}

\author{
Bonan Yan \\ Duke University \\ Durham, North Carolina \\ bonan.yan@duke.edu
}

\author{
Xiong Cao \\ Duke University \\ Durham, North Carolina \\ xiong.cao@duke.edu
}

\author{
Hai (Helen) Li \\ Duke University \\ Durham, North Carolina \\ hai.li@duke.edu
}

\begin{abstract}
The recent proposed nanoscale Mott memristor features negative differential resistance and chaotic dynamics. This work proposes a novel neuromorphic computing system that utilizes Mott memristors to simplify peripheral circuitry. According to the analytic description of chaotic dynamics and relaxation oscillation, we carefully tune the working point of Mott memristors to balance the chaotic behavior weighing testing accuracy and training efficiency. Compared with conventional designs, the proposed design accelerates the training by $1.893 \times$ averagely and saves $27.68 \%$ and $43.32 \%$ power consumption with $36.67 \%$ and $26.75 \%$ less area for singlelayer and two-layer perceptrons, respectively.
\end{abstract}

\section{CCS CONCEPTS}

- Hardware $\rightarrow$ Analysis and design of emerging devices and systems;

\section{KEYWORDS}

Mott memristor, neuromorphic computing system

\section{ACM Reference Format:}

Bonan Yan, Xiong Cao, and Hai (Helen) Li. 2018. A Neuromorphic Design Using Chaotic Mott Memristor with Relaxation Oscillation. In $D A C$ '18: DAC '18:The 55th Annual Design Automation Conference 2018, June 24-29, 2018, San Francisco, CA, USA. ACM, New York, NY, USA, 6 pages. https: //doi.org/10.1145/3195970.3195977

\section{INTRODUCTION}

Neuromorphic computing system (NCS) has been under intensive investigations to response the ever-increasing demands of machine learning applications [1-3]. In NCS deployment, emerging nonvolatile memories (eNVMs) that feature high storage density and power efficiency have shown great potential. Particularly, the memristor (a.k.a. resistive memory or ReRAM) technology provides multiple (or analog) resistance levels to represent synaptic weights and the high-resistance range for low power consumption. The memristor crossbar array structure naturally realize matrix-vector multiplications [4], enabling an efficient processing-in-memory (PIM) solution. For example, Alibart et al. [5] used a memristor crossbar to

Permission to make digital or hard copies of all or part of this work for personal or classroom use is granted without fee provided that copies are not made or distributed for profit or commercial advantage and that copies bear this notice and the full citation on the first page. Copyrights for components of this work owned by others than ACM must be honored. Abstracting with credit is permitted. To copy otherwise, or republish, to post on servers or to redistribute to lists, requires prior specific permission and/or a fee. Request permissions from permissions@acm.org.

DAC '18, fune 24-29, 2018, San Francisco, CA, USA

(C) 2018 Association for Computing Machinery.

ACM ISBN 978-1-4503-5700-5/18/06 .. \$15.00

https://doi.org/10.1145/3195970.3195977 mimic a single-layer perceptron network for pattern classification. Spiking-based NCS implementations have also been demonstrated, many of which use integrate-and-fire circuit (IFC) as the output neuron $[6,7]$. Although the adoption of memristor arrays dramatically improves computing and storage efficiency, the neuron circuitry largely dominates the power consumption and design area. For example, the IFC circuit in [6] consists of 15 transistors and 1 capacitor and occupies an area of $175.3 \mu^{2}$ at GlobalFoundries $130 \mathrm{~nm}$ technology node. Moreover, as the analog implementation is usually adopted to realize the neuron function, further simplifying the CMOS-based neuron design is difficult.

Pickett et al. presented Mott memristor in 2013 [8]. The simple device demonstrates the properties of neuron and therefore is used to built neuristor. Furthermore, the recent experiments by HPE labs showed that Mott memristor brings quasi-randomness to output owing to its chaotic dynamics [9]. This property helps training escape from local minima throughout the back-propagation stage and consequently accelerate the network training procedure. Although the preliminary use of Mott memristor as neuristor alone with temporal coding [8] and together with memristor arrays [9] have been presented, it lacks explicit study on how to use Mott memristor to construct NCS. Moreover, although the chaotic dynamics feature of Mott memristor is beneficial to training procedure, it may degrades the computation accuracy during the recall (inference) procedure. How to balance the chaotic dynamics in training and recall needs further investigation.

To address these problems, our work studies the neuromorphic design that integrates memristor crossbar and neuristors built with Mott memristors. Compared to the prior research, the innovations of this work reflect in the following two aspects:

- A novel neuron design is proposed. Our work uses Mott memristor, combined with a 7-transistor amplifier, as the neuron circuit. Moreover, the detailed design of the entire NCS implementation based on Mott memristor is demonstrated.

- The working regions (domains) of Mott memristor are calibrated quantitatively. The target is to take advantage of chaotic behavior of Mott memristor in training and simultaneously maintains a relatively high recall accuracy.

We implemented and simulated the proposed neuromorphic design based on the $\mathrm{TaO}_{\mathrm{x}}$ memristor model from [10], the Mott memristor model from [11], and GlobalFoundries 130 $\mathrm{nm}$ CMOS technology. The designs in $[6,12,13]$ are taken as the baseline. For single-layer and two-layer designs [14], our proposed design saves $27.68 \%$ and $43.32 \%$ power consumption with $36.67 \%$ and $26.75 \%$ less area, respectively. Moreover, we evaluate the training efficiency of our design by comparing it with the feed-forward network in [5]. 


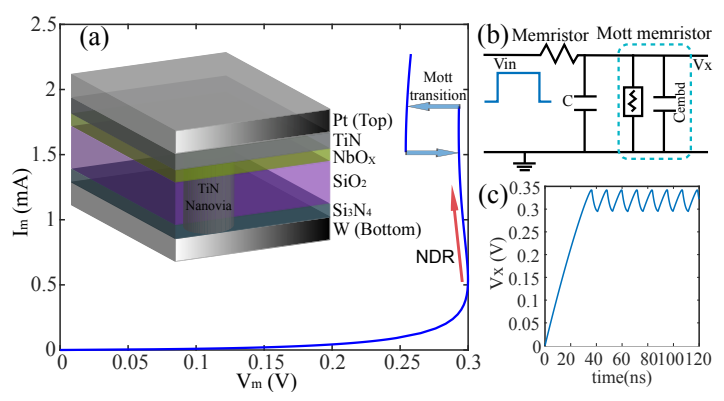

Figure 1: (a) The device structure and static I-V characteristics of Mott memristor; (b) the schematic of Pearson-Anson relaxation oscillation circuit with Mott memristor; (c) the output sawtooth-shape waveform of the relaxation oscillation circuit due to forced oscillation.

The training steps reduce $1.893 \times$ owing to the chaotic dynamics of Mott memristor.

\section{PRELIMINARIES}

Memristor is a two-terminal non-linear passive component relating to electrical charge and magnetic flux. The existence of memristor was predicted by Leon Chua in 1971 [15]. Till 2008 a team at $\mathrm{HP}$ Labs connected the operation of $\mathrm{a}_{\mathrm{TiO}} \mathrm{x}$ device to the memristor concept [16]. The resistance of a memristor, also known as memristance, reflects the history of current flowing through it. By adjusting the amplitude and pulse width of programming voltage/current, a memristor can be programmed to multiple discrete resistance levels or continuous resistance states [10]. The two-terminal structure of a memristor device enables the construction of an ultra-dense crossbar array for data storage or computation, in which a memristor is allocated at each crosspoint of horizontal and vertical metal wires $[4,17]$

Mott memristor. Figure 1(a) depicts the device structure and the static current-voltage (I-V) characteristics of Mott memristor. The device has a reversible metal-insulator phase transition [18] at a specific temperature $\left(T_{C}\right)$ and thus demonstrates a history-related resistance. $\mathrm{NbO}_{2}$ Mott memristor shows the current-controlled negative differential resistance (NDR) due to a thermal feedback mechanism, which has been proved both theoretically and experimentally [11]. As shown in Figure 1(a), NDR appears when the amplitude of the current flowing through the device is within the range between $0.6 \mathrm{~mA}$ and $1.3 \mathrm{~mA}$ : the voltage across the Mott memristor becomes smaller as the applied current increases. In the NDR region, Mott memristors have chaotic characteristics that randomly perturb the I$\mathrm{V}$ characteristics of Mott memristor [9]. A recent study showed that a Pearson-Anson relaxation oscillation circuit $[19,20]$ integrated with a $\mathrm{NbO}_{2}$ Mott memristor demonstrates chaotic oscillation [9]. Figure 1(b) depicts the circuit diagram and the corresponding simulation result for the oscillation is presented in Figure 1(c). Chaotic oscillation means that the amplitude and frequency of the oscillation have slight random difference over time. This phenomenon is caused by thermal fluctuation of Mott memristor that acts as an intrinsic oscillator.

Memristor-based NCS design. Because of the high similarity between memristor device and biological synapse, the use of memristors in NCS has been intensively studied. The crossbar structure is particularly attractive for the implementation of synaptic connections and denotes as the connection matrix in neural network models. By adjusting the amplitude and pulse width of programming voltage/current, a memristor can be programmed to multiple discrete resistance levels or continuous resistance states to represent a synaptic weight in neural network models. The design concept has been investigated and demonstrated, offering a new design scenario with high computation efficiency [5, 21, 22].

Although the adoption of memristor arrays dramatically improves computing and storage efficiency in these designs, the neuron circuit determines the performance of NCS's, including area, power, recognition accuracy, and training speed. For example, the voltage-based sensing scheme in [4] requires analog-digital and digital-analog converters (ADC/DAC), which results in large signal distortion and power consumption. The spiking based designs commonly adopt integrate-and-fire circuit (IFC) to generate output spikes. Although the power efficiency dramatically improves, an IFC still requires a number of transistors and at least one capacitor for charge accumulation [6]. It is very difficult to further reduce the design complexity and layout area of the CMOS based implementations within this scheme. Instead, we build a novel neuron circuit based on the modified Pearson-Anson relaxation oscillation circuit with Mott memristor.

\section{DESIGN OVERVIEW \& PRINCIPLE}

The proposed NCS includes the memristor crossbar array and Mott memristor neuron circuits. These two parts and their integrated equivalent circuits are presented in Figure 2. In the design, the computation will be executed on a $m \times n$ memristor crossbar array. A set of input signals $V_{i}^{i n}(i=1,2, \cdots, m)$ are used to denote the input vector and supplied to the crossbar array along bitlines (BLs). The induced current across memristors are accumulated along sourcelines (SLs). Our proposed neuron circuit built with Mott memristor is connected to each SL and produce output spikes.

The relationship between the input signals, memristances and output of neuron circuit is the operating principle of the proposed system. For each crossbar array column, the equivalent circuit in Figure 2(b) shows that the current flowing through the $j^{\text {th }}$ column

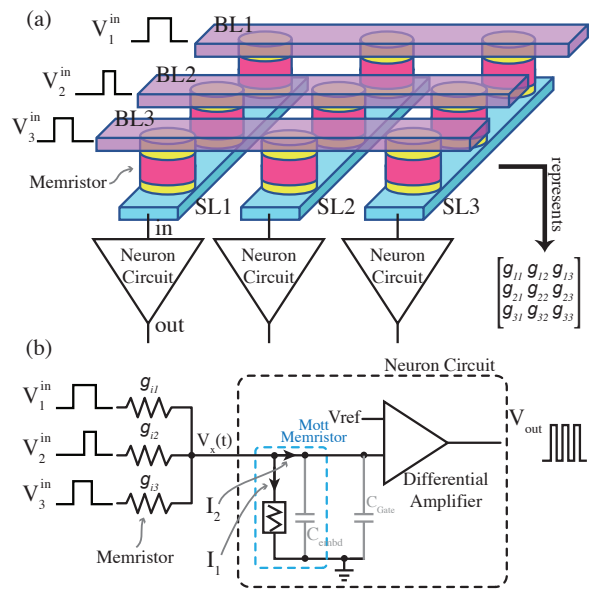

Figure 2: The circuit diagram of the proposed design (a) and the equivalent circuit (b). 
Table 1: Mott memristor modeling parameters

\begin{tabular}{c|c|c}
\hline Variable & Physical Meaning & Value \\
\hline$A$ & Active area of $\mathrm{NbO}_{\mathrm{x}}$ & $\pi(70 \mathrm{~nm} / 2)^{2}$ \\
$\sigma_{0}$ & Material constant & $8.4 \times 10^{4} \mathrm{~A}^{2} \mathrm{sN}^{-1} \mathrm{~m}^{-2}$ \\
$k_{B}$ & Boltzmann constant & $1.38 \times 10^{-23} \mathrm{~m}^{2} \mathrm{kgs}^{-2} \mathrm{~K}^{-1}$ \\
$\omega$ & Material constant & $2.59 \times 10^{-24} \mathrm{C}^{\frac{1}{2}} \mathrm{NC}^{\frac{1}{2}} \mathrm{~m}$ \\
$d$ & Thickness of NbO $\mathrm{x}$ & $8 \mathrm{~nm}$ \\
$T_{a m b}$ & Ambient temperature & $300 \mathrm{~K}$ \\
$T_{C}$ & Mott transition temperature & $1,070 \mathrm{~K}$ \\
$C_{t h}$ & Thermal capacitance & $10^{-16} \mathrm{WsK}^{-1}$ \\
$R_{t h}(T)$ & Effective thermal resistance & $1.4 \times 10^{6} \mathrm{~kW}^{-1}, T<T_{C}$ \\
& & $2.0 \times 10^{6} \mathrm{~kW}^{-1}, T>T_{C}$ \\
\hline
\end{tabular}

follows the relation according to Kirchhoff's law:

$$
\sum_{i=1}^{m}\left[\left(V_{i}^{i n}-V_{x}(t)\right) \cdot g_{i j}\right]=I_{1}+I_{2},
$$

where $V_{i}^{i n}$ is the input voltage to the $i^{\text {th }}$ row of the memristor crossbar, $V_{x}(t)$ is a time-variant voltage at the input of the neuron circuit, and $g_{i j}$ denotes the conductance of the cell across the $i^{\text {th }}$ row and the $j^{\text {th }}$ column of the memristor crossbar. The first term on the left-hand side of Equation (1) is the multiplication results of two vectors, i.e., $\sum V_{i}^{i n} g_{i j}=\mathrm{V}^{i n} \cdot \mathrm{G}_{j} . I_{1}$ and $I_{2}$ on the right side correspond to the currents flowing through the Mott memristor and its shunt capacitor, respectively.

$I_{1}$ is determined by both the Mott memristor characteristics and the electrical excitation condition, i.e., $V_{x}(t)$ in this design. The conductance of a Mott memristor $g_{\text {Mot } t}(t)$ depends on the evolution of its intrinsic temperature $T$ [9]. So we have:

$$
\begin{aligned}
I_{1} & =V_{x}(t) \cdot g_{\text {Mot } t}, \\
g_{\text {Mot } t} & =g_{\text {Mott }}\left(V_{x}(t), T\right) \\
& =A \sigma_{0} \mathrm{e}^{-\frac{0.301}{2 k_{B} T}}\left\{\left(\frac{k_{B} T}{\omega}\right)^{2}\left[1+\left(\frac{\omega \sqrt{\frac{V_{x}(t)}{d}}}{k_{B} T}-1\right) \frac{\omega \sqrt{\frac{V_{x}(t)}{d}}}{k_{B} T}\right]+\frac{1}{2 d}\right\}, \\
\frac{\mathrm{d} T}{\mathrm{~d} t} & =\frac{\mathrm{d} T}{\mathrm{~d} t}\left(V_{x}(t), T\right) \\
& =\frac{V_{x}^{2}}{C_{t h} g_{\text {Mott }}}-\frac{T-T_{a m b}}{C_{t h} R_{t h}(T)}+T \sqrt{\frac{k_{B}}{C_{t h}}} \cdot \frac{(2 \mathrm{~b})}{R_{t h}(T) C_{t h} \cos \left(\frac{2 \pi t}{\left(R_{t h}(T) C_{t h}\right.}\right)} .
\end{aligned}
$$

Table 1 summarizes the physical meanings and values of the parameters used in Equation (2), which are collected from references $[9,11]$.
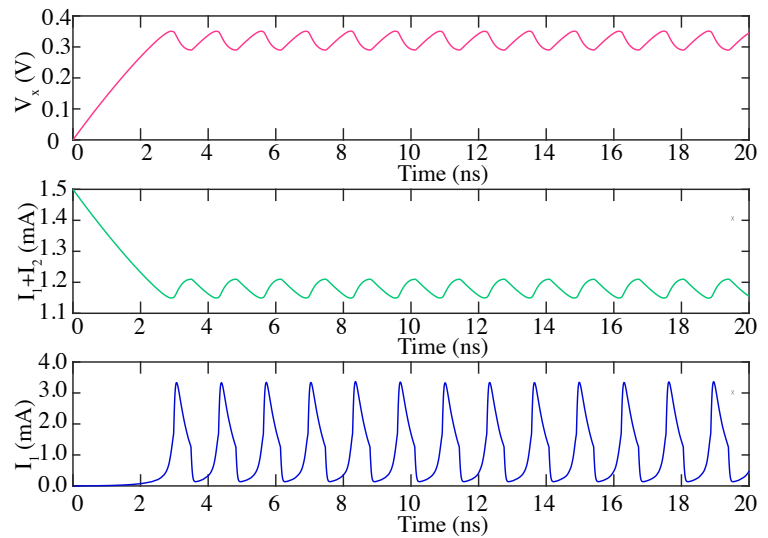

Figure 3: The numerical solution of relaxation oscillation.
$I_{2}$ is the alternating current in the shunt capacitor. It can be written in a generalized form of the differential equation of charging and discharging of capacitor.

$$
I_{2}=C \frac{\mathrm{d} V_{x}(t)}{\mathrm{d} t},
$$

where $C=C_{e m b d}+C_{\text {Gate }}$ is the total shunt capacitance parallel with the Mott memristor. $C_{e m b d}$ is the capacitance embedded into the Mott memristor device and $C_{\text {Gate }}$ is the parasitic input capacitance of the proceeding amplifier.

The numerical solutions by combining Equations (1-3) shown in Figure 3. This solution is obtained when the effective conductance of memristor crossbar array column is $\sum g_{i j}=1 \mathrm{mS}$ and the input voltage is $1.2 \mathrm{~V}$. This figure presents the voltage oscillation on the node $V_{x}(t)$, the current oscillations in the SL branch $\left(I_{1}+I_{2}\right)$ and the Mott memristor branch only $\left(I_{1}\right)$. The large amplitude of $I_{1}$ indicates the extensive charge exchange between the Mott memristor and the shunt capacitor. The creation of the sawtooth wave is due to the combined force of the NDR effect and the rapid memristance change induced by the Mott transition.

Different from previous capacitor charging based IFC neuron circuit designs $[6,13]$, the oscillation waveform is sawtooth shape instead of exponential or linear. Its oscillation frequency is the output of Mott memristor neuron circuit. Technically, the oscillation is guaranteed by Poincaré-Bendixson theorem.

Theorem 3.1. There exists a periodic solution of $V_{x}(t)$ oscillating and its voltage amplitude is in the range of $\left[\frac{\mathrm{V}^{i n} \cdot \mathrm{G}_{j}}{g_{\text {Mot }}^{\max }+\sum_{i=1}^{m} g_{i j}}, \frac{\mathrm{V}^{\text {in }} \cdot \mathrm{G}_{j}}{g_{\text {Mott }}^{\text {min }}+\sum_{i=1}^{m} g_{i j}}\right]$, where $g_{\text {Mott }}^{\max }$ and $g_{\text {Mott }}^{\text {min }}$ are the maximum and minimum solutions of $\mathbf{V}^{i n} \cdot \mathrm{G}_{j}=\left(g_{M o t t}+\sum_{i=1}^{m} g_{i j}\right) V_{x}(t)$, respectively.

Proof. Equation (1) can be written as:

$$
\begin{aligned}
& \sum_{i=1}^{m}\left[\left(V_{i}^{i n}-V_{x}(t)\right) \cdot g_{i j}\right]=g_{M o t t} V_{x}(t)+C \frac{\mathrm{d} V_{x}(t)}{\mathrm{d} t} \\
& \Leftrightarrow \frac{\mathrm{d} V_{x}(t)}{\mathrm{d} t}=\frac{1}{C}\left[\mathrm{~V}^{i n} \cdot \mathrm{G}_{j}-\left(g_{M o t t}+\sum_{i=1}^{m} g_{i j}\right) V_{x}(t)\right] .
\end{aligned}
$$

According to Poincaré-Bendixson theorem [23], if $\frac{\mathrm{d} V_{x}(t)}{\mathrm{d} t}$ does not keep the same sign in the given range, the solution of $V_{x}(t)$ is periodic. When $\frac{\mathrm{d} V_{x}(t)}{\mathrm{d} t}=0$, the differential equation has only one stationary solution, if exists. Hence, the initial condition leads to the trivial stationary solution. Otherwise (the case that $\frac{\mathrm{d} V_{x}(t)}{\mathrm{d} t}$ is not always 0 ), as $V_{x}(t)$ evolves, $g_{M o t}$ changes accordingly, owing to the dependency of $g_{\text {Mott }}$ on $V_{x}(t)$. The highest and lowest range of $g_{\text {Mot } t}$ must make $V_{x}(t)$ reaches extrema: $\frac{\mathrm{d} V_{x}(t)}{\mathrm{d} t}=0$. Including this condition into Equation (4) results in a transcendental equation: $0=\mathrm{V}^{i n} \cdot \mathrm{G}_{j}-\left(g_{M o t}+\sum_{i=1}^{m} g_{i j}\right) V_{x}(t)$. There exists at least two solutions because of the the bifurcation of Mott memristor due to Mott transition and NDR as discussed in Section 2. Considering the convexity, Equation (4) must be positively and negatively approaching the two extrema. Thus $V_{x}(t)$ has periodic solution and it oscillates in the range the two extrema range.

Proof is completed. 


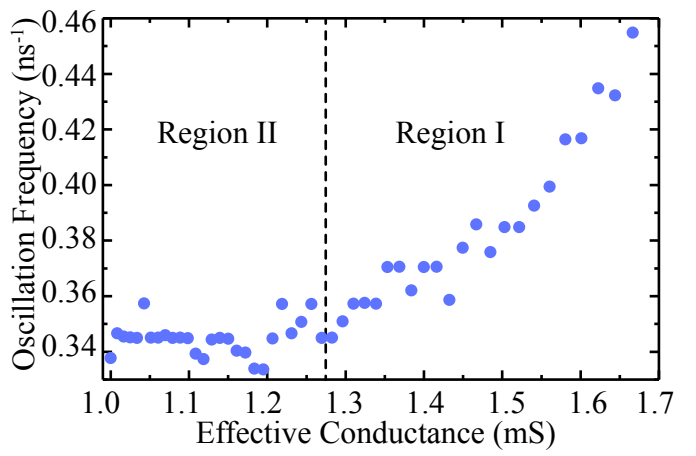

Figure 4: The relationship between the effective conductance and the output pulse frequency.

THEOREM 3.1 provides the confinement of the oscillating voltage amplitude, guiding the design of post-signal amplifier. Unfortunately, we cannot derive an analytic solution of the oscillation period (frequency) from these nonlinear transcendental equations. Nevertheless, we used numerical algorithm to obtain the relationship of the output frequency and the effective conductance of a column of memristors $\left(\sum g_{i j}\right)$, which is shown in Figure 4 . We divide the results into two regions. Region I is a quasi-linear region which can be used to convert the output current of memristors into output spikes, in which domain our proposed design works. In Region II where $\sum_{1}^{m} g_{i j}<1.27 m S$, the chaotic dynamic becomes more obvious as the thermal-induced nonlinear charge exchanging between the capacitor and Mott memristor dominates the output.

\section{HARDWARE DESIGN}

\subsection{Memristor and Mott Memristor}

In our design, two types of emerging devices are used for the different purposes. Memristors construct the crossbar array for matrix multiplication; Mott memristors build the Pearson-Anson circuit, which converts the current from SLs into output spikes.

In the memristor crossbar design, a selective device is serially connected to memristor in each cell to avoid the sneak path during programming. The selector in each cell makes the overall cell resistance (the memristance together with the selector resistance) sensitive to the effective voltage applied to the cell. As such, the analogy of memristor crossbar to the matrix multiplication also becomes sensitive to the input signals. Take 1-Transistor-1-Memristor (1T1M) cell design as an example, Figure 5 shows the impact of SL voltage on the overall cell resistance. The results is obtained based on the condition by assuming the input (BL) voltage of $1.2 \mathrm{~V}$. In the figure, the $x$-axis represents the SL voltage $V_{S L}$. Then the effective voltage applied to a target cell is $V_{c e l l}=1.2 \mathrm{~V}-V_{S L}$. The $y$-axis denotes the cell effective resistance, which is the sum of memristance and the drain-source resistance of the transistor.

In the IFC-based design [6], the SL voltage changes from $0 \mathrm{~V}$ to $0.6 \mathrm{~V}$, inducing approximate $40.81 \%$ of cell resistance changing. To avoid the large cell resistance change, an additional current amplifier [13] is proposed to stabilize SL voltage. However, it induces high power and area cost. In contrast, the SL voltage oscillates in the range of $0.242 \mathrm{~V}$ to $0.376 \mathrm{~V}$ in our proposed design, which leads to a negligible $0.61 \%$ cell resistance variation. However, the output

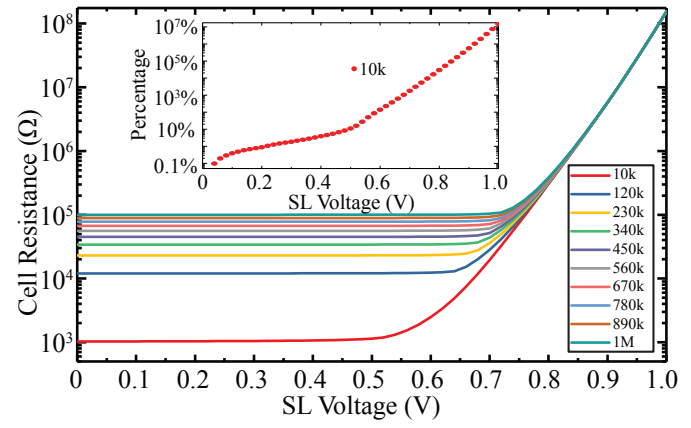

Figure 5: The impact of SL voltage on the cell resistance. The inset figure: the worst-case cell resistance changes in percentage vs. SL voltage.

voltage has a small margin, which requires an output buffer to amplify the signal.

\subsection{Neuron Circuit}

Figure 6 shows the diagram of the neuron circuit and waveforms of internal nodes. In addition to the revised Pearson Anson relaxation oscillation circuit with Mott memristor, we include an output buffer to amplify $V_{x}(t)$ and reshape it to digital output spikes. Confined by NDR ratio of Mott memristor, $V_{x}(t)$ is a biased triangle wave like signals with small amplitude of approximate $0.1 \mathrm{~V}$. The output buffer shall guarantee the $V_{x}(t)$ oscillation crossing the input high-low threshold to ensure this analog signal can be converted into digital spikes. Aiming to minimize the design area, we use a combination of a differential amplifier (M1 - M5) and an inverter (M6 - M7). The former magnifies the signals to make sure the oscillating $V_{x}(t)$ cross the high-low input threshold of the proceeding inverter and increase the sensing margin of $V_{x}(t)$. And the latter serves as a signal regulator and shapes the amplified $V_{x}(t)$ to binary signals.

$V_{x}(t)$ is fed to the gate of M1. A reference voltage $V_{r e f}$ is connected to the gate of M2 as the threshold to digitalizes $V_{x}(t)$. M5 serves as the current sink to match the single-ended output bias. Figure 6(b) shows the waveforms of $V_{x}(t), V_{\text {mid }}$-amplified $V_{x}(t)$, and the final binary output spikes $V_{\text {out }}$. Note that the phase of output spikes does not affect computing accuracy because the calculation results is modulated to the frequency.

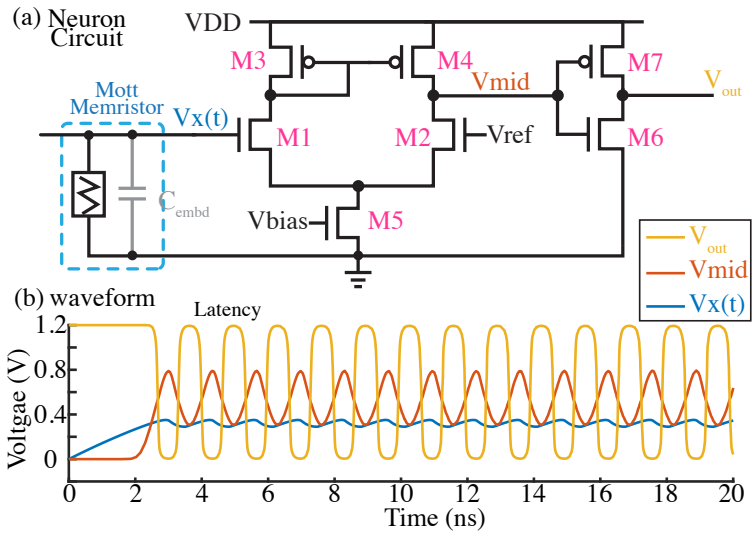

Figure 6: (a) The diagram of the proposed neuron circuit; (b) the waveforms of $V_{o u t}, V_{\text {mid }}$ and $V_{x}(t)$. 

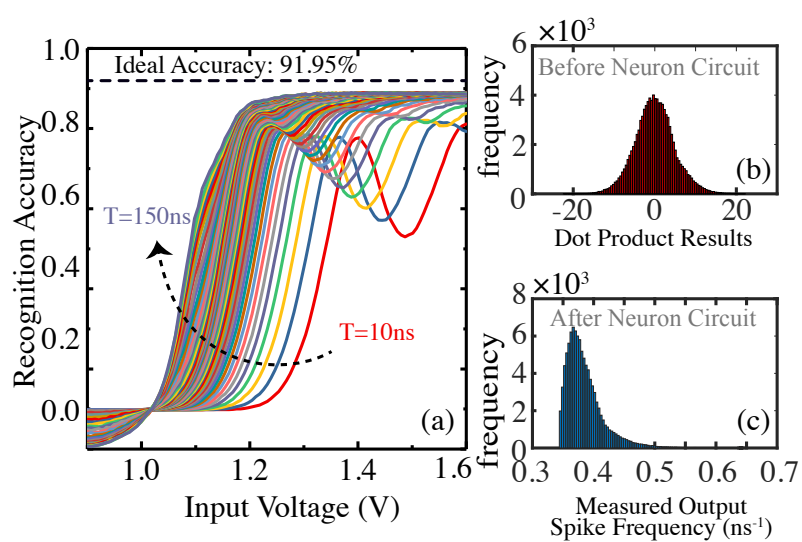

Figure 7: (a) Recall accuracy vs. input voltage; (b) the distribution of vector-vector multiplication at software level; (c) the distribution of measured output spike frequencies.

\subsection{Chaotic Dynamics in Recall \& Training}

In a pattern recognition application, for instance, the largest element in the output vector indicates which class the input pattern belongs to. If random noise is added on neuron circuits, the original largest element might goes below than the others, resulting in classification errors. The chaotic dynamics of Mott memristor is equivalent to inject random noises to the neuron circuit and therefore the output vector of a one-layer network design. As shown in Figure 4, as the effective conductance grows larger, higher current forces the Mott memristor to approach the stationary point, weakening the thermal impact in the oscillation. The output spike frequency is likely to be more stable. Because the input information is coded as the pulse number, we are able to control the chaotic dynamics by changing input voltage amplitude. In the recall operation or the feed-forward stage of the training operation, the input voltage shall be enlarged to suppress the chaotic dynamics.

In contrast, the randomness is beneficial to speed up the training. The alleged "training plateau" describes the condition stuck in local minimums, which prevents or slows down the convergence in training by confining updated weights to zero. The randomness, however, can help to get out of local minimums and therefore speed up the training. By tunning input voltage, the randomness is controlled only in the feed-forward phase of the training operation. Each training epoch can be divided into three steps: (1) apply a low voltage, e.g., $1.0 \mathrm{~V}$, to carry out the feed-forward phase with chaotic dynamics; (2) calculate update weights according to the output spikes (with randomness) and input pattern; and (3) update the crossbar with a high programming voltage, e.g., $3.0 \mathrm{~V}$.

\section{DESIGN EVALUATION}

\subsection{Simulation Setup}

In this work, we use single-layer and two-layer perceptrons to recognize the handwritten digits in MNIST dataset as the example applications. The recall recognition accuracy of $91.95 \%$ for singlelayer perceptron and $95.23 \%$ for two-layer perceptron are obtained from the software level training using the stochastic gradient descent approach. The activation functions in the example neural networks are Relu. The number of hidden units (HU) in the two-layer perceptron ranges from 50 to 300 to represent different structures of neural networks. Moreover, for the circuit level verification, we adopt $130 \mathrm{~nm}$ CMOS technology with $V d d=1.2 \mathrm{~V}$. The cell of memristor crossbar array is 1-Transistor-1-Memristor (1T1R) structure.

\subsection{Recalling}

We evaluate the impact of chaotic dynamics in the recall operations by observing the voltage dependent recognition accuracy of the proposed design, which is shown in Figure 7(a). These curves are obtained by sweeping the recall latency $T$ from $10 n$ s to $150 \mathrm{~ns}$ with a step of $2.86 n$ s. As the input voltage increases, the recognition accuracy generally improves because of more linearity obtained. Larger recall latency indicates less digitalization error as more output spikes are captured. The accuracy "well” (accuracy drops first and rises again as input voltage increases) emerges when $V^{\text {in }} \in$ $[1.4,1.6]$ and $T=10 n s$, which is caused by both digitalization error and chaotic dynamics. Digitalization error dominates when latency is too short $(<100 n s)$ to collect enough output spikes to achieve sufficient accuracy. As the recall latency increases, the accuracy well vanishes because digitalization error is not as critical. Instead, chaotic dynamics become more dominant.

Figure 7(b) presents the histogram of the vector-vector multiplication for 1000 test data, at software level. Except for a scaling coefficient, these values are given as the input into the neuron circuits. Figure 7 (c) is obtained with a recall latency of $150 \mathrm{~ns}$ and at $V^{i n}=1.2 \mathrm{~V}$. A distorted distribution of the output spikes is observed. It implies that the relationship in Figure 4 is not completely linear, reflecting the randomness Mott memristor brings.

\subsection{Training}

In the evaluation of training operations, the mean square error (MSE) is used as the error (cost) function. Figure 8 shows the comparison of MSE evolution in training between the cases with and without (w/o) chaotic dynamics. The chaotic oscillation is realized by applying a lower voltage ( $1.1 \mathrm{~V}$ in this experiment) in the feedforward stage of training operation. We compare the MSE descending rate in the given range of training epochs. In the example applications, chaotic dynamics boosts training speed by $1.893 \times$ on average because the quasi-randomness helps training escape from local minima [24].

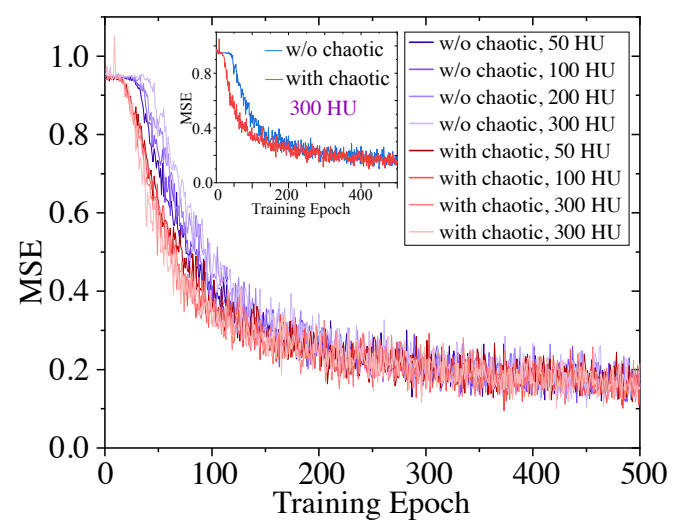

Figure 8: The MSE evolution in training. The training is accelerated by the chaotic dynamics. The inset figure magnifies the pair of curves for the $300 \mathrm{HU}$ cases. 
Table 2: System performance comparison

\begin{tabular}{c||c|c||c|c}
\hline Design & Baseline/Single-layer [6, 13] & This work/single-layer & Baseline/Two-layer [12, 13] & This work/two-layer \\
\hline Neuron circuit & $1 \mathrm{C}+15 \mathrm{~T}$ & $1 \mathrm{Mott}+7 \mathrm{~T}$ & $1 \mathrm{C}+15 \mathrm{~T}$ & $1 \mathrm{Mott}+7 \mathrm{~T}$ \\
Area & $0.00649 \mathrm{~mm}^{2}$ & $0.00411 \mathrm{~mm}^{2}$ & $0.0114 \mathrm{~mm}^{2}$ & $0.00835 \mathrm{~mm}^{2}$ \\
Power & $1.12 \mathrm{~mW}$ & $0.81 \mathrm{~mW}$ & $11.45 \mathrm{~mW}$ & $6.49 \mathrm{~mW}$ \\
Recall latency & $100 \mathrm{~ns}$ & $100 \mathrm{~ns}$ & $200 \mathrm{~ns}$ & $200 \mathrm{~ns}$ \\
Energy/recall & $111.77 \mathrm{fJ}$ & $81.23 \mathrm{fJ}$ & $2290.60 \mathrm{fJ}$ & $1298.11 \mathrm{fJ}$ \\
\hline
\end{tabular}

Because Mott memristors only exist after each crossbar array (weight matrix), different HU numbers represent the number of Mott memristor based neuron circuits. As indicated in Figure 8, the larger HU number, indicating more neuron circuits, results in larger difference between the cases of with and without chaotic dynamics.

\subsection{Power and Area}

We compare the overall performance of the proposed design and the baseline designs $[6,12,13]$. Table 2 summarizes the comparisons of specifications including circuit complexity, area, power, recall latency and energy. The target design of two-layer network has 300 hidden units. In this table, "C", "T" and "Mott" represent capacitor, transistor and Mott memristor, respectively.

Specifically, the recall latency of the proposed design represents the computing period to collect output spikes. According to Figure 7, $100 \mathrm{~ns}$ output latency per layer are employed to maintain the highest accuracy when $V^{i n}=1.2 \mathrm{~V}$, which is the same computing speed with implementations in $[6,12,13]$. The speed of output spike generation of our design, according to Equation (4), relies heavily on the embedded capacitance C. An optimized Mott memristor device with reduced embedded capacitor results in higher speed.

The energy consumption denotes the total energy required to finish the entire recognition procedure. Mott memristor based neuron circuits bring $27.68 \%$ and $43.32 \%$ less power consumption in single-layer and two-layer design, respectively. As the dimension of neural network rises, the numbers of both memristors and neuron circuits increase. Because neuron circuits dominate in power consumption, more power consumption reduction is expected as the network dimension grows.

Circuit complexity is reflected by the number of components. The proposed design reduces 8 transistors and 1 capacitors in each neuron circuit. The deeper neural network is, the more neuron circuits appear in the computing system. Our work saves $36.67 \%$ and $26.75 \%$ area for single-layer and two-layer implementations, respectively. The percentage reduction from $36.67 \%$ and $26.75 \%$ as the network dimension rises because the increment of memristor numbers (weight numbers) is larger than that of neuron circuits.

\section{CONCLUSION}

In this paper, we present a neuromorphic design using chaotic Mott memristor to simply the neuron circuits, aiming at low power consumption and high density. The design of Mott memristor based neuron circuit is guided by the theorem according to our analysis. By calibrating the input voltage, the chaotic dynamics are suppressed in recall to promise accurate calculation while excited in training procedure. By exploiting Mott memristor and reduce circuit complexity, the proposed methodology consumes over $26 \%$ less power and area compared with the conventional designs to recognize handwritten digits as single-layer and two-layer perceptron.

\section{ACKNOWLEDGMENT}

This research is supported by NSF CCF-1725456 and NSF CSR1717885 .

\section{REFERENCES}

[1] N. P. Jouppi and et al. 2017. In-datacenter performance analysis of a tensor processing unit. In International Symposium on Computer Architecture. 1-12.

[2] Y.-H. Chen and et al. 2017. Eyeriss: An energy-efficient reconfigurable accelerator for deep convolutional neural networks. IEEE fournal of Solid-State Circuits 52, 1 (2017), 127-138.

[3] P. N. Whatmough and et al. 2017. $14.3 \mathrm{~A} 28 \mathrm{~nm}$ SoC with a $1.2 \mathrm{GHz}$ $568 \mathrm{~nJ} /$ prediction sparse deep-neural-network engine with $>0.1$ timing error rate tolerance for IoT applications. In International Solid-State Circuits Conference. 242-243.

[4] M. Hu and et al. 2012. Hardware Realization of BSB Recall Function Using Memristor Crossbar Arrays. In Design Automation Conference. 498-503.

[5] F. Alibart and et al. 2013. Pattern classification by memristive crossbar circuits using ex situ and in situ training. Nature communications 4 (2013).

[6] C. Liu and et al. 2015. A spiking neuromorphic design with resistive crossbar. In Design Automation Conference. 1-6.

[7] S. Yu. 2018. Neuro-Inspired Computing With Emerging Nonvolatile Memorys. Proc. IEEE 106, 2 (2018), 260-285.

[8] M. D. Pickett and et al. 2013. A scalable neuristor built with Mott memristors. Nature materials 12, 2 (2013), 114-117.

[9] S. Kumar and et al. 2017. Chaotic dynamics in nanoscale NbO2 Mott memristors for analogue computing. Nature 548, 7667 (2017), 318-321.

[10] J. P. Strachan and et al. 2013. State dynamics and modeling of tantalum oxide memristors. IEEE Transactions on Electron Devices 60, 7 (2013), 2194-2202.

[11] G. A. Gibson and et al. 2016. An accurate locally active memristor model for S-type negative differential resistance in NbOx. Applied Physics Letters 108, 2 (2016), 023505.

[12] B. Yan and et al. 2017. A closed-loop design to enhance weight stability of memristor based neural network chips. In Proceedings of the 36th International Conference on Computer-Aided Design. IEEE Press, 541-548.

[13] C. Liu and et al. 2016. A memristor crossbar based computing engine optimized for high speed and accuracy. In Computer Society Annual Symposium on VLSI. 110-115.

[14] Y. LeCun and et al. 1998. Gradient-based learning applied to document recognition. Proc. IEEE 86, 11 (1998), 2278-2324.

[15] L. Chua. 1971. Memristor-the missing circuit element. IEEE Transactions on circuit theory 18, 5 (1971), 507-519.

[16] D. B. Strukov and et al. 2008. The missing memristor found. nature 453, 7191 (2008), 80-83.

[17] J. J. Yang and et al. 2013. Memristive devices for computing. Nature nanotechnology 8,1 (2013), 13-24.

[18] F. Chudnovskii and et al. 1996. Electroforming and switching in oxides of transition metals: The role of metal-insulator transition in the switching mechanism. fournal of Solid State Chemistry 122, 1 (1996), 95-99.

[19] P. SO and et al. 1921. The neon tube as a means of producing intermittent currents. (1921).

[20] S. Pearson and et al. 1921. Demonstration of some electrical properties of neonfilled lamps. Proceedings of the Physical Society of London 34, 1 (1921), 175.

[21] M. Hu and et al. 2016. Dot-product engine for neuromorphic computing: programming 1T1M crossbar to accelerate matrix-vector multiplication. In Design Automation Conference. 1-6.

[22] F. M. Bayat and et al. 2016. Advancing Memristive Analog Neuromorphic Networks: Increasing Complexity, and Coping with Imperfect Hardware Components. arXiv preprint arXiv:1611.04465 (2016).

[23] A. J. Schwartz. 1963. A generalization of a Poincaré-Bendixson theorem to closed two-dimensional manifolds. American fournal of Mathematics (1963), 453-458.

[24] L. Dinh and et al. 2017. Sharp minima can generalize for deep nets. arXiv:1703.04933 (2017) 